\title{
The Ship Integration Design Platform Architecture Based on IPDE
}

\author{
Huang Jinfeng \\ China Ship Development and Design Center, Wuhan 430064, China \\ howardhjf@163.com
}

Keywords: Ship, Integration Design, Platform Architecture, IPDE

\begin{abstract}
The US Navy Product Data Standard defines a set of requirements for an effective IPDE, focusing primarily on the traditional data management capabilities and interfaces with $\mathrm{CAD} / \mathrm{CAM} / \mathrm{CAE} / \mathrm{CAO}, \mathrm{PDM}, \mathrm{ERP}$ and catalog systems used for the design, construction and in-service support of Navy ships. The purpose of the paper is to set up an ship integration design platform, especially in the areas of configuration and change management, and the interoperability of systems within an IPDE and between disparate IPDEs; so being to drive down the cost of change and increase the reuse of ship product data over the entire ship's life cycle.
\end{abstract}

\section{Introduction}

The design and construction of a class of Navy ships can span a decade all by itself. The information required to support this effort is voluminous and constantly changing. History indicates that the advent of computer technology to the shipbuilding process has been a double-edged sword bringing advances in process automation as well as reductions in design cycle time, design errors found during construction, the number of procured parts, and the number of Navy drawing approvals. It has eliminated the need for physical mock-ups, and many other labor-intensive design and development practices albeit at the price of increased complexity in information management. Managing change and obtaining efficient information interoperability have risen to become major cost drivers in the shipbuilding industry.

An Integrated Product Data Environment (IPDE) is a collection of business processes, computer systems, and associated services, which house the product model data, and enable people to work in concert towards common business goals throughout the life cycle of a product. Designing and maintaining a ship using an IPDE can add significant efficiencies to the process, but if not done wisely, it can also add unacceptable cost and risk to a shipbuilding Program[1].

The paper will set up an ship design development environment based on IPDE, and provide a synopsis of the IPDE strategy as implemented.

\section{Architecture of IPDE}

\section{is The Navy Ship Integration Design Platform Boundaries on IPDE}

This paper focuses on requirements for the core capabilities of the navy ship integration design platform on IPDE along with requirements for Interoperability and Data Exchange between the major applications that make up an IPDE. Fig. 1 illustrates the boundary of the navy ship integration design platform[1]. This notional model shows the applications supporting the major shipbuilding and support functions as vertical columns. These vertical applications are different for each implementation of an IPDE; some IPDE's will not have all the applications or include additional applications. The IPDE core capabilities support the vertical applications and provide management and control access to the product model data. 


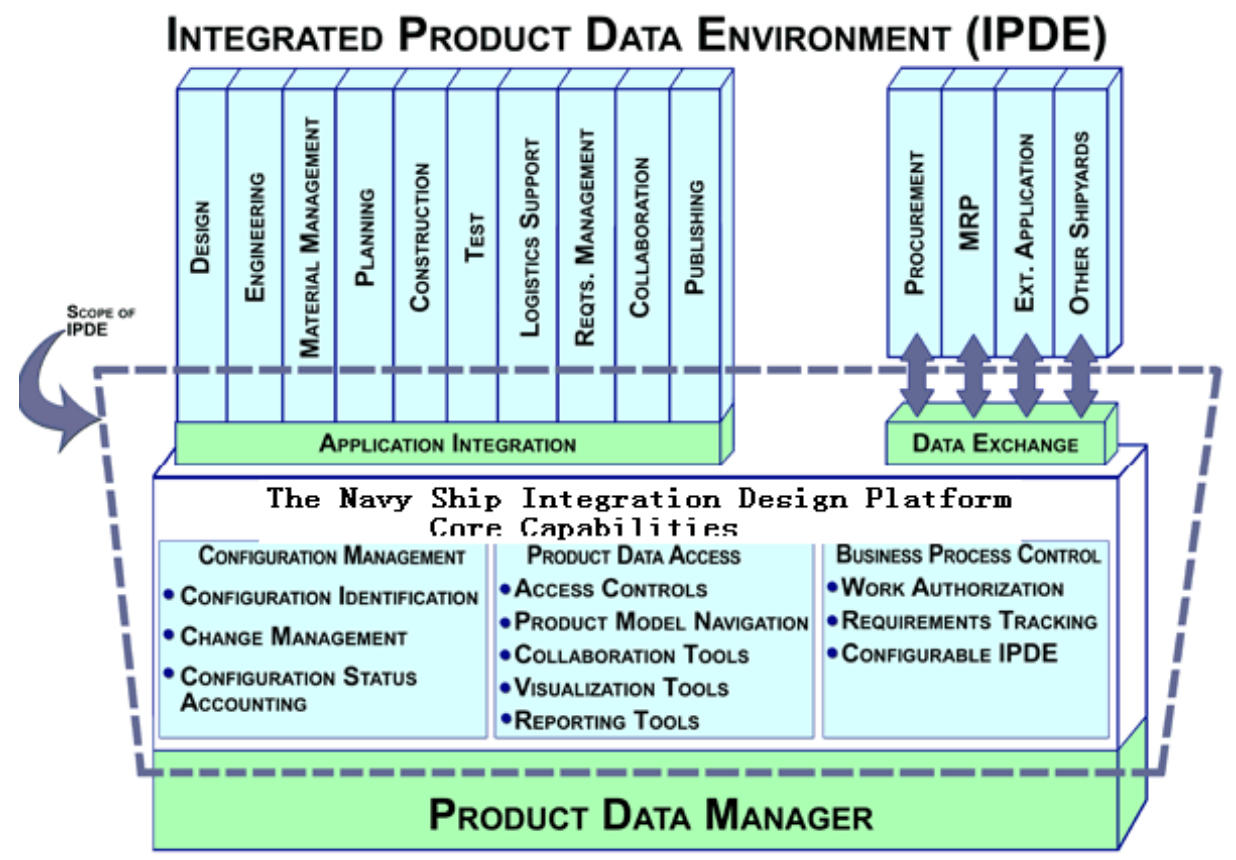

Fig.1 The Navy ship integration design platform boundaries on IPDE

\section{is Shipbuilding Needs and the IPDE}

The life cycle of a class of ships can be 30 to 40 years and the IPDE needs to be designed to support the product data throughout the life cycle.

These needs are organized according to the different shipbuilding disciplines:Design/Engineering, Material Management, Planning, Construction and Test, Integrated Logistics Support, Requirements Management, Collaboration, Reporting and Publishing.

Fig.2 illustrates the navy ship integration design platform's role on IPDE in supporting the product data over the life cycle of a class of ships and the transfer of that data from one IPDE to another. The figure shows the progression of life cycle phases, from Concept Definition through Construction and Test, In-Service Support(including Ship alts), and finally Decommissioning. During the design and construction phases,the figure shows that there is typically a set of PDM and application tools that capture and maintain the product data in a native format. However at any time during those phases, product data may need to be transferred to external systems or archived. As shown in the figure, this transfer would comply with the Ship Common Information Model (SCIM). Once a ship is in service, product data may be transferred to other contractor's IPDEs or other Navy Systems[2].

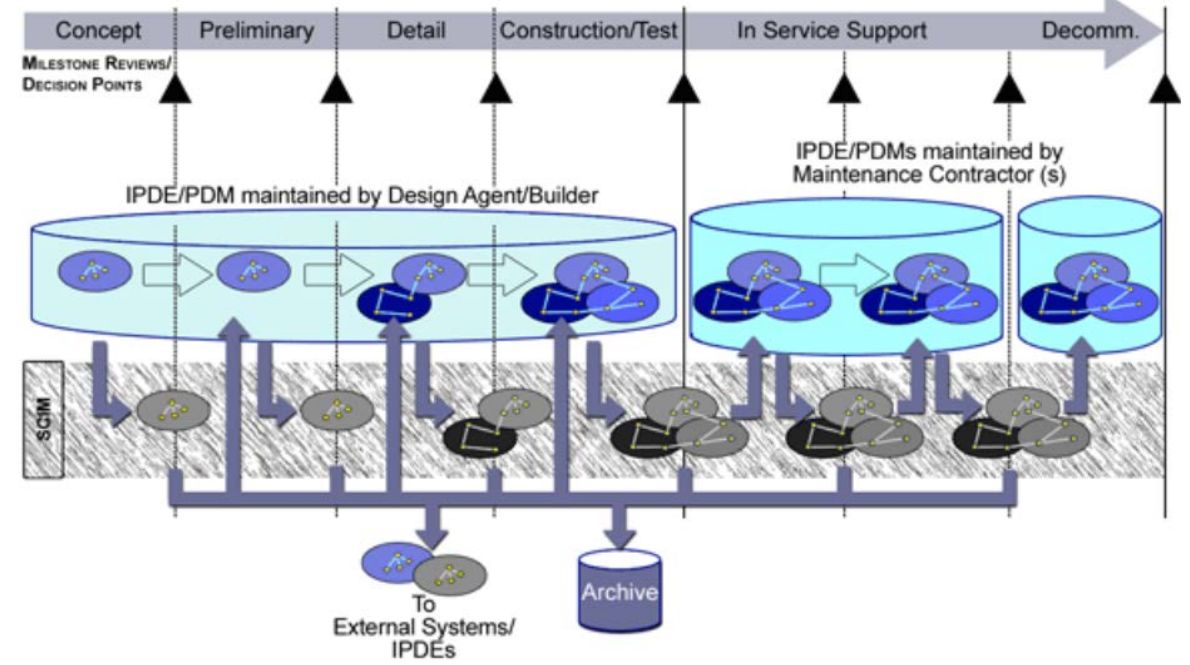

Fig.2 The navy ship integration design platform needs to transition throughout the life cycle 


\section{is Core Capabilities of the Navy Ship Integration Design Platform}

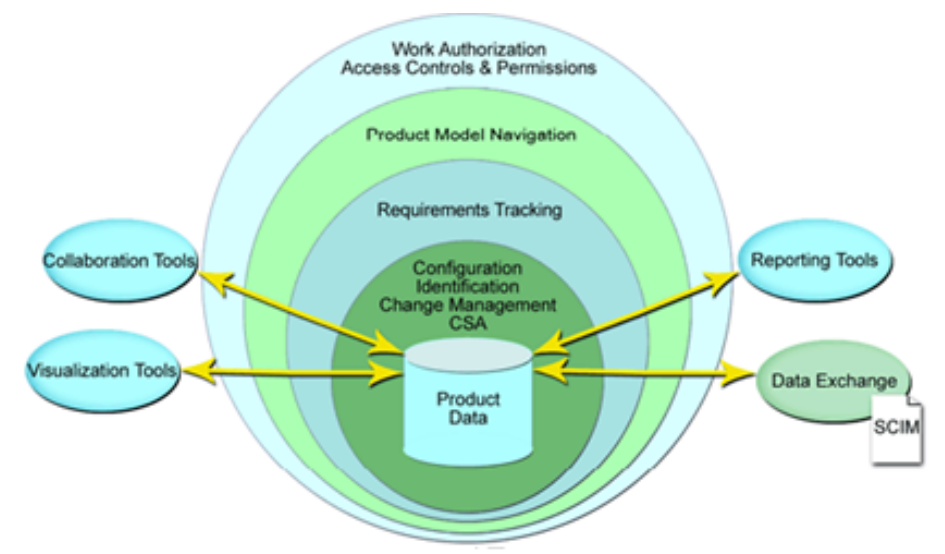

The Navy Ship Integration Design Platform on IPDE

Fig.3 The navy ship integration design platform core on IPDE and cross cutting capabilities

The core capabilities listed in Fig.3 represent a set of functionality that need to be addressed by an IPDE implementation to effectively manage ship product model data over the life cycle of a Navy vessel.Figure 3 shows a notional relationship between these core capabilities. Some of this functionality may already be incorporated into existing commercial Product Data Managers, but it is the integration of these capabilities that creates the most cost-effective functionality for the IPDE. Specific requirements are described in Section 3.

Surrounding the Product Data is a set of capabilities that maintain, control, and protect the data from corruption or misuse. At the heart of the system is Configuration Management which is designed to manage all the shipbuilding product data elements. This capability includes Configuration Identification, Change Management and Configuration Status Accounting. Requirements Tracking maintains linkages to program and product requirements as changes are identified and implemented. Product Model Navigation provides the tools to find and select the parts of the Product Model relevant to a particular user's needs without locking data unnecessarily. Access Control and Permissions protect the IPDE from unauthorized user activity and Work Authorization integrates with the Shipyard business processes to ensure the proper accounting for work done with the Product Model.

\section{Core Capability Requirements}

This section, along with next section, contains the enforceable IPDE requirements. These requirements are derived from the Shipbuilding Needs described in above section and address the core Product Data capabilities of an IPDE. These core capabilities, depicted in Fig.4, are organized into three main areas: configuration management, product data access, and business process control[3].

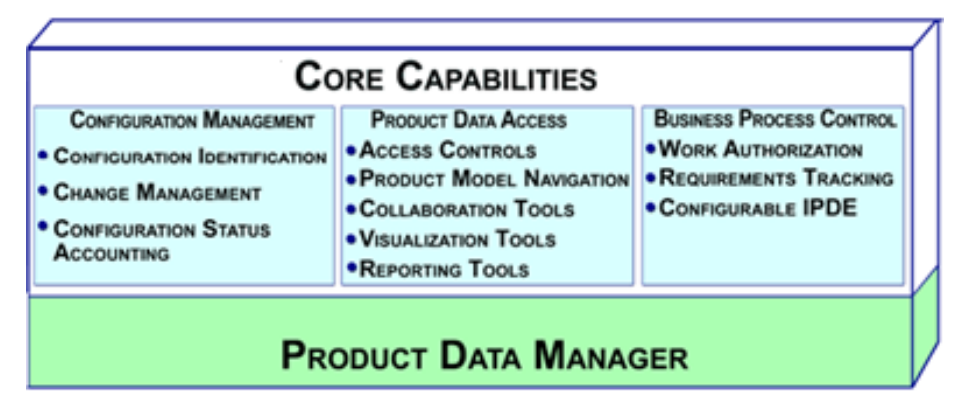

Fig.4 Core capabilities

It is not expected that these requirements will be satisfied by one software tool, rather it is anticipated that there may be several tools, whether they are commercial, government, or custom shipyard-specific tools, that are integrated or interfaced to provide a shipyard-specific 
implementation of these requirements. The relationship between the IPDE core capabilities and the tools which implement them is depicted in Fig.4. In this figure the IPDE core capabilities are satisfied by a combination of COTS, as well as, Custom Tools. The specific allocation of software requirements to tools or applications will be shipyard-specific. For example, suppose that Tool C is the PDM tool used in a shipyard. The entire tool is within the shipyard IPDE, but it only implements a portion of the core IPDE capability defined in this paper. Tool B may provide the additional PDM capabilities required by this specification.These tools may also provide additional PDM capabilities that may be used as part of the overall shipyard IPDE implementation. This additional functionality may be utilized within the shipyard,but it is not part of the core IPDE capabilities specified in this document. Another point to note from Fig.5 is that there may be overlap between functionality provided by one tool and functionality provided by another tool. Again, how this functionality is integrated or utilized within a specific instance of an IPDE depends on the needs of the shipyard and/or program.

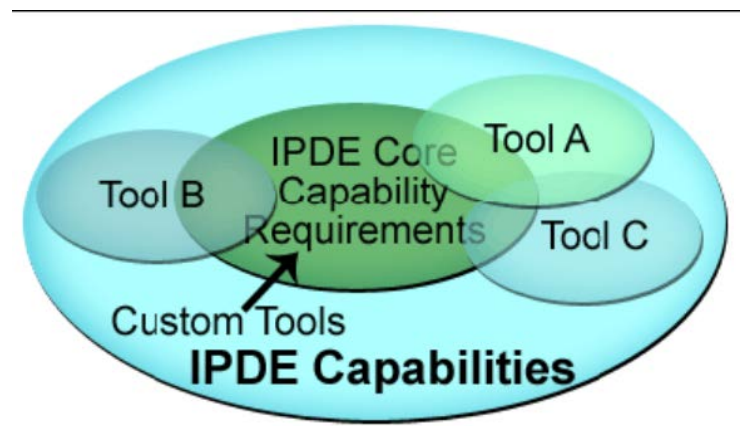

Fig.5 Notional IPDE requirements allocation

\section{Interoperability Requirements}

There are two main elements of interoperability: technical and operational.The technical aspects of interoperability deal mainly with the definition of data and interface specifications, along with the means with which two or more entities communicate. Operational interoperability is more procedural in nature; its aspects concern the ability of a system to enable effective and efficient collaboration. Although shipbuilding IPDEs need to enable interoperability for both to the greatest extent possible this section will focus on the technical aspects by discussing application integration and data exchange.

\section{is Ship Common Information Model (SCIM)}

The Ship Common Information Model (SCIM) document defines the minimum information thatmust be maintained within an IPDE and the format needed to exchange it to enable effective interoperability among shipbuilding IPDEs. It is intended to be used throughout a typical shipbuilding life cycle, and is designed to be invoked and mandated on future contracts[4]. 


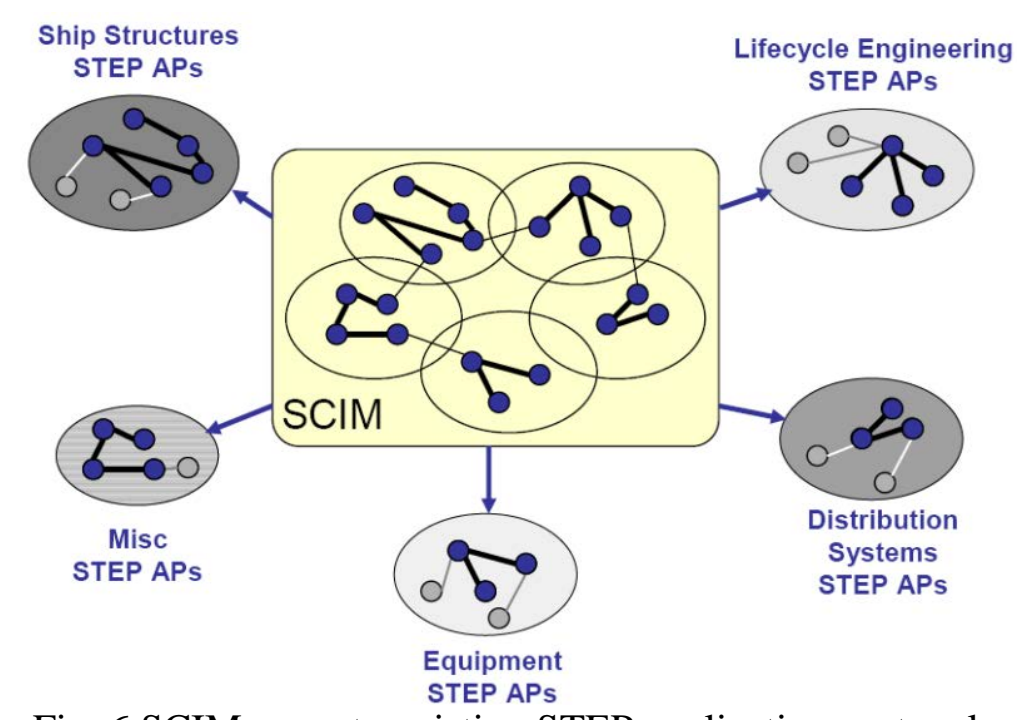

Fig. 6 SCIM maps to existing STEP application protocols

The SCIM is based on several existing STEP Application Protocol (AP) Standards (ISO 10303) and the schemas developed under the NSRP's Integrated Shipbuilding Environment (ISE) Projects for the various shipbuilding domains[5]. SCIM-compliant files will be in XML format. Schema elements from these STEP standards, that are relevant to the shipbuilding domain, have been identified and organized as one coherent product model schema. The purpose is to define a single, common data vocabulary to be referenced by all future shipbuilding IPDEs for data exchange and application integration. Fig.6demonstrates that the SCIM addresses application areas defined in several different STEP Application Protocols (APs).

\section{is IPDE Interoperability Requirements}

\section{Product Data Accessibility.}

The IPDE shall have the capability to export, import, and query product data as defined by the SCIM.The IPDE shall be able to import product data that is defined in the SCIM, and must be able to ignore any additional data within the SCIM that may not be required for a particular application.The IPDE shall have standardized interface and query mechanisms to access Product Data.

\section{Data Exchange.}

The IPDE shall be able to produce a SCIM-compliant XML file to represent the product model data in each application area specified in the SCIM that is supported by the IPDE. SCIM compliant XML means that there exists a mapping to and from the XML schema defined by the SCIM such that there is no loss of information in a transformation. This mapping should be able to be defined completely using X Path/XSLT or other compatible technologies.

\section{Data Exchange Formats/Protocols.}

The ability to efficiently move data across applications in the same domain, applications within the same IPDE, or applications within separate IPDEs may require:Integration, Data Sharing, Migration, Design in context, Consistent semantics and format.

\section{Mediators.}

If the XML files supported by the IPDEs involved are not in a standard neutral file format (such as STEP Part 28) then Figure 4-4 does not apply and the SCIM exchange becomes more complicated. The actual representations themselves used by each vendor may in fact be different(different names, different distribution of properties among entities, etc.). A "Mediator" will be required to translate from the XML file generated by the sending IPDE to the XML format that can be read by the receiving IPDE[6]. 


\section{Summary}

The paper will address the need to enable system interoperability within a company's IPDE or across disparate IPDEs in a collaborative nature. There are two aspects to interoperability; Open Systems and Data Exchange. Open Systems addresses the need for an open IPDE architecture. Data Exchange deals first with the scope and content of the data to be transferred and second, the methods of transfer.

\section{References}

[1] Navy Product Data InitiativeIntegrated Product Data Environment(IPDE) Specification. 2008,7,30.

[2] Howard Fireman,Marianne Nutting etc. LPD17 ON THE SHIPBUILDING FRONTIER: INTEGRATED PRODUCTION \&PROCESS DEVELOPMENT.35 ${ }^{\text {th }}$ Annual Technical Symposium.1998.04.

[3] Open Architecture (OA) Computing Environment Technologies and Standards Version1.0 23 August 2004.

[4] National Shipbuilding Research Program (NSRP) Navy Product Data Initiative (NPDI) Ship Common Information Model(SCIM), Deliverable under Task of NSRP ASE Project Technology Investment Agreement (TIA)\#2010-627.

[5] Usage Guide for the STEP PDM Schema V1.2, Release 4.3.

[6] Steel Processing Final Report, Document Number ISE-4-STEEL-0004, Version 1.0, NSRP ASE ISE-4 Technology Investment Agreement (TIA) 2005-381, Integrated Shipbuilding Environment Consortium (ISEC). 